\title{
Calcium-Independent, cGMP-Mediated Light Adaptation in Invertebrate Ciliary Photoreceptors
}

\author{
Maria del Pilar Gomez ${ }^{1,2}$ and Enrico Nasi ${ }^{1,2}$ \\ ${ }^{1}$ Department of Physiology and Biophysics, Boston University School of Medicine, Boston, Massachusetts 02118, and ${ }^{2}$ Marine Biological Laboratory, \\ Woods Hole, Massachusetts 02543
}

Calcium is thought to be essential for adaptation of sensory receptor cells. However, the transduction cascade of hyperpolarizing, ciliary photoreceptors of the scallop does not use $\mathrm{IP}_{3}$-mediated Ca release, and the light-sensitive conductance is not measurably permeable to $\mathrm{Ca}^{2+}$. Therefore, two typical mechanisms that couple the light response to $[\mathrm{Ca}]_{\mathrm{i}}$ changes seem to be lacking in these photoreceptors. Using fluorescent indicators, we determined that, unlike in their microvillar counterparts, photostimulation of ciliary cells under voltage clamp indeed evokes no detectable change in cytosolic Ca. Notwithstanding, these cells exhibit all of the hallmarks of light adaptation, including response range compression, sensitivity shift, and photoresponse acceleration. A possible mediator of Ca-independent sensory adaptation is cGMP, the second messenger that regulates the light-sensitive conductance; cGMP and 8-bromo cGMP not only activate lightdependent $\mathrm{K}$ channels but also reduce the amplitude of the light response to an extent greatly in excess of that expected from simple occlusion between light and chemical stimulation. In addition, these substances accelerate the time course of the photocurrent. Tests with pharmacological antagonists suggest that protein kinase $\mathrm{G}$ may be a downstream effector that controls, in part, the cGMP-triggered changes in photoresponse properties during light adaptation. However, additional messengers are likely to be implicated, especially in the regulation of response kinetics. These observations suggest a novel feedback inhibition pathway for signaling sensory adaptation.

Key words: photoreceptors; light adaptation; photosensitivity; cyclic nucleotides; sensory transduction; calcium

\section{Introduction}

The process of light adaptation enables the visual system to respond optimally over a wide range of stimulating conditions without incurring saturation. Intense scrutiny of the cellular and molecular mechanisms that mediate adaptation in different classes of photoreceptors have pointed to a pivotal role for "intracellular" calcium because of two important characteristics: (1) its ability to affect key enzymatic steps of the phototransduction cascade and (2) the fact that its concentration is regulated by light stimulation. In the case of rods and cones, illumination leads to a reduction of cytosolic [Ca] (Yau and Nakatani, 1985; McNaughton et al., 1986; Ratto et al., 1988) by decreasing the influx through light-sensitive channels (Yau and Nakatani, 1984a) in the face of a sustained extrusion by the $\mathrm{Na} / \mathrm{Ca} / \mathrm{K}$ exchanger (Yau and Nakatani, 1984b). Calcium influences the transduction cascade of vertebrate photoreceptors at multiple sites (for review, see Fain et al., 2001). In the microvillar photoreceptors of invertebrates, $[\mathrm{Ca}]_{\mathrm{i}}$ increases during photostimulation (Brown and Blinks, 1974), and this elevation is a key factor in light adaptation (Lisman and Brown, 1972, 1975; Bader et al., 1976; Fein and Charlton, 1977). The mechanisms by which Ca participates in light adaptation of rhabdomeric photoreceptors are not com-

Received March 11, 2004; revised Jan. 10, 2005; accepted Jan. 12, 2005.

This work was supported by National Institutes of Health Grant R01-EY07559.

Correspondence should be addressed to Dr. Maria del Pilar Gomez, Department of Physiology and Biophysics,

Boston University School of Medicine, 715 Albany Street, Boston, MA 02118. E-mail: mpgomez@bu.edu.

D01:10.1523/JNEUROSCI.5129-04.2005

Copyright $\odot 2005$ Society for Neuroscience $\quad$ 0270-6474/05/252042-08\$15.00/0 pletely understood but include feedback inhibition of the $\mathrm{IP}_{3}$ receptor (Payne et al., 1990) and stimulation of a protein kinase $C$ (PKC) (Smith et al., 1991; Hardie et al., 1993; Piccoli et al., 2002).

Not all photoreceptors found in invertebrate organisms are microvillar. The eyes of several marine mollusks also contain ciliary photoreceptors that respond to light with a hyperpolarization of the membrane potential (for review, see McReynolds, 1976). Furthermore, at least in the bay scallop Pecten irradians, such as in vertebrate photoreceptors, cGMP serves as a second messenger for phototransduction. Nonetheless, there are some key differences that place these ciliary receptors in a novel category, separate from rods and cones. (1) They do not express transducin $\left(G_{t}\right)$; instead, the only detectable heterotrimeric G-protein is a $G_{o}$ (Kojima et al., 1997), and pharmacological data are consistent with its involvement in light responsiveness (Gomez and Nasi, 2000). (2) The light-induced hyperpolarization is attributable to an increase of a K-selective conductance (Gorman and McReynolds, 1978; Cornwall and Gorman, 1983; Gomez and Nasi, 1994) rather than the closure of nonselective channels. (3) As a consequence, the photoresponse calls for an elevation of cGMP, and previous studies (Gomez and Nasi, 2000) suggest that the light-regulated enzyme is a guanylate cyclase, as opposed to a phosphodiesterase (PDE).

In sharp contrast with microvillar photoreceptors, no significant involvement of the phospholipase $\mathrm{C}$ (PLC)/ $\mathrm{IP}_{3} / \mathrm{Ca}$ cascade has been detected in these cells (Gomez and Nasi, 1995). This creates a unique situation among photoreceptors, in that the two most widespread mechanisms normally used to couple light 
stimulation to changes in cytosolic calcium (namely influx through light-dependent channels and release from internal stores) are not operative. Nevertheless, all of the manifestations of light adaptation are present in these cells, and this process is essentially impervious to exogenous manipulations of calcium (Gomez and Nasi, 1997). This indicates the existence of a novel set of mechanisms capable of modulating the light response. In the present report, we addressed the nature of the signaling pathway that governs adaptation in a Ca-independent manner. Our observations reveal the presence of a novel feedback mechanism that regulates the sensitivity and kinetics of the light response and is controlled by cGMP, the same messenger that gates the lightsensitive conductance.

Parts of this work have been published previously in abstract form (Gomez and Nasi, 1999).

\section{Materials and Methods}

Single-cell electrophysiology. All of the experiments were conducted on specimens of $P$. irradians obtained from the Aquatic Resources Division of the Marine Biological Laboratory. The protocols to dissociate the retina were described previously (Gomez and Nasi, 1994). Whole-cell currents were recorded from isolated ciliary photoreceptors plated onto a recording chamber continuously perfused with artificial sea water (ASW) containing the following (in $\mathrm{mM}$ ): $480 \mathrm{NaCl}, 10 \mathrm{KCl}, 10 \mathrm{CaCl}_{2}, 49$ $\mathrm{MgCl}_{2}, 10 \mathrm{HEPES}$, and 5.5 D-glucose, $\mathrm{pH}$ 7.75. Patch electrodes fabricated with thin-walled borosilicate capillary tubing (type 7052; Garner Glass, Claremont, CA) pulled to a $2-3 \mu \mathrm{m}$ outer tip diameter were fire polished and filled with an intracellular solution containing the following: $100 \mathrm{~mm} \mathrm{KCl}, 200 \mathrm{~mm} \mathrm{~K}$-glutamate, $22 \mathrm{~mm} \mathrm{NaCl}, 5 \mathrm{~mm} \mathrm{Mg}$ ATP, 10 mM HEPES, 1 mм EGTA, $100 \mu \mathrm{M}$ GTP, and 300 mм sucrose, pH 7.3 (electrode resistance, 2-4 M $\Omega$ in ASW). Series resistance was compensated via a positive feedback circuit in the amplifier (maximal residual error typically $<2 \mathrm{mV}$ ). Currents were low-pass filtered with a Bessel four-pole filter, using a cutoff frequency of $1.5-2 \mathrm{kHz}$, and digitized at a sampling rate of $3-5 \mathrm{kHz}$ by a 12-bit resolution analog-to-digital (A/D) interface board (model 2821; Data Translation, Marlboro, MA). Light responses are measured with respect to the baseline current; because the cells were voltage clamped at $-30 \mathrm{mV}$, close to their resting potential, this holding current was close to zero. Voltage and light stimuli were applied by a microprocessor-controlled programmable stimulator (Stim 6; IonOptix, Milton, MA).

Chemical stimulation. cAMP and the slowly hydrolyzable cyclic nucleotide analogs 8-bromo cGMP (8-Br-cGMP) and 8-Br-cAMP were purchased from Sigma (St. Louis, MO). Cilostamide, an inhibitor of type III PDE (PDE III), and forskolin were also from Sigma. The PKG inhibitors KT5823 and RQIKIWFQNRRMKWKK-LRK ${ }_{5} \mathrm{H}$ (DT-3) were from Calbiochem (San Diego, CA). The PKG pseudosubstrate heptapeptide G-1458 was obtained from LC Laboratories (Woburn, MA). The Rp isomer of cAMP (Rp cAMP), a membrane-permeable, hydrolysisresistant inhibitor of cAMP-dependent PKA, was obtained from Biolog Life Science Institute (Bremen, Germany). Intracellular application of test substances was performed by dissolving them in the internal solution and dialyzing them via the patch pipette. Extracellular application entailed ejection from a micropipette (tip diameter, $3-4 \mu \mathrm{m}$ ) positioned $\sim 50 \mu \mathrm{m}$ from the target cell. The puffer pipette was connected to a solenoid-operated valve and a precision regulator, which served to apply pressurized nitrogen.

Light stimulation. Two optical stimulators were used as described previously (Gomez and Nasi, 1997). One used a $100 \mathrm{~W}$ tungsten-halogen light source (Oriel, Stratford, CT), and its output beam was combined with that of the microscope illuminator via a beam-splitter prism placed above the condenser. In the second stimulator, the source was a $100 \mathrm{~W}$ mercury arc lamp (Zeiss, Oberkochen, Germany), and light was delivered via a liquid light guide (Oriel) to the epifluorescence port of the inverted microscope, so that it reached the preparation via the microscope objective. In both cases, in front of the condenser, there was an infrared absorbing filter, an electromechanical shutter (Vincent Associ- ates, Rochester, NY), and collimating and field lenses. An adjustable, pinhole, or an iris diaphragm placed in a conjugated image plane restricted the illuminated region on the recording chamber to a disc $\sim 200$ $\mu \mathrm{m}$ in diameter. Broadband light was used in all of the experiments (515-670 nm), determined by the combination of a heat-absorbing filter and an edge filter (Schott Glass Technologies, Duryea, PA) interposed in the light path. Calibrated neutral-density filters (Melles Griot, Irvine, CA) provided controlled light attenuation. During experimental manipulations, the cells were viewed with a Newvicon television camera (model WV-1550; Panasonic, Secaucus, NJ) using a near infrared long-pass filter for illumination $(\lambda>780 \mathrm{~nm}$; Andover, Salem, NH). The infrared illuminator was turned off for several minutes before light responses were tested.

Intracellular calcium monitoring. To measure Ca fluorescence, the excitation light from a $75 \mathrm{~W}$ xenon arc source (Photon Technology International, Lawrenceville, NJ) was reflected off of a cold mirror $\left(\lambda_{\mathrm{c}}=670\right.$ $\mathrm{nm}$; Omega, Brattleboro, VT), passed through an interference filter $\left(\lambda_{\max }=480 \mathrm{~nm}\right.$; bandwidth, $40 \mathrm{~nm}$; Chroma Technology, Brattleboro, VT), and fed to the epi-illumination port of the inverted microscope via a liquid light guide (Oriel). Emission light was collected by a 100×, 1.3 numerical aperture oil-immersion objective (Nikon, Tokyo, Japan); the filter block in the turret contained a $505 \mathrm{~nm}$ dichroic reflector and a barrier filter $\left(\lambda_{\max }=535 \mathrm{~nm}\right.$; bandwidth, $50 \mathrm{~nm}$; Chroma Technology). At the camera port, an adjustable, positionable mask (Nikon) was used to circumscribe the collected fluorescence to a defined rectangular region of interest (usually the light-transducing region of the isolated photoreceptor), to minimize background light. The output light was split further by an additional dichroic mirror $\left(\lambda_{\mathrm{c}}=610 \mathrm{~nm}\right)$, which diverted the fluorescence signal to a photomultiplier tube (PMT) (model R4220 PHA; Hamamatsu, Bridgewater, NJ), which was operated at $800 \mathrm{~V}$ and connected to a preamplifier-discriminator and a photon counter (models F-100T and PRM-100; Advanced Research Instrumentation, Boulder, $\mathrm{CO})$. An analog voltage proportional to the counts accumulated in bins of programmable duration (typically, $10^{-5} \mathrm{sec}$ ) was fed to the A/D interface of the computer. Long-wavelength light was directed to the infrared-sensitive camera used to visualize the cells. Electromechanical shutters (Vincent Associates) controlled excitation light and PMT exposure. Photoreceptors were loaded with the fluorescent $\mathrm{Ca}^{2+}$ indicators Calcium Green-2 or Calcium Green-5N (Molecular Probes, Eugene, $\mathrm{OR}$ ); the potassium salt of the probe was dissolved in the intracellular solution filling the patch electrode at a concentration of 30-85 $\mu \mathrm{M}$. Before testing, $\approx 4-6 \mathrm{~min}$ of internal dialysis time was allowed to elapse.

\section{Results}

In view of the previously reported ineffectiveness of $\mathrm{IP}_{3}$ administration and the lack of measurable Ca permeation via the photoconductance in ciliary photoreceptors, we first set out to directly monitor cellular free calcium to ascertain whether lightdependent changes may occur via alternative mechanisms. We determined previously that light sensitivity only began to be depressed with applied $[\mathrm{Ca}]_{\mathrm{i}}$ in the $10^{-5} \mathrm{M}$ range (Gomez and Nasi, 1997). Therefore, we selected Calcium Green-5N as the fluorescent indicator, reasoning that its high $K_{\mathrm{D}}(14 \mu \mathrm{M})$ would be appropriate for reporting a light-evoked $\mathrm{Ca}^{2+}$ elevation in a range that could be functionally significant. The indicator was loaded into voltage-clamped ciliary photoreceptors via the patch electrode in the whole-cell configuration. After $\approx 4$ min of internal dialysis, the epi-illumination beam from a xenon arc source was turned on for $500 \mathrm{~ms}$, and the fluorescence collected from a $\approx 10 \times 10 \mu \mathrm{m}$ region (which included the light-sensitive ciliary appendages of the cell) was measured simultaneously with the membrane current. In Figure 1, the top trace shows the outward photocurrent elicited by the excitation light beam, whereas the bottom trace shows the emitted fluorescence measured by the PMT. The optical signal jumped instantaneously after the shutter was opened, reflecting the basal level of light emission, but no additional change in fluorescence occurred during the light re- 


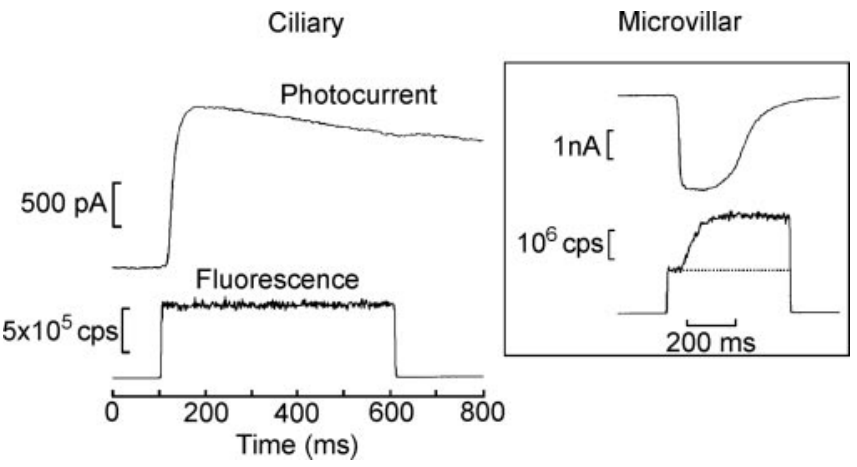

Figure 1. The light response is not accompanied by changes in cytosolic calcium. Simultaneous recording of membrane current and fluorescence in a voltage-clamped ciliary photoreceptor. The cell was dialyzed for $\approx 4$ min with an internal solution containing the $\mathrm{Ca}^{2+}$ indicator Calcium Green-2 $(83 \mu \mathrm{M})$. The epi-illumination beam from a xenon arc source was turned on for $500 \mathrm{~ms}$ ( $\lambda_{\max }=480 \mathrm{~nm}$; bandwidth, $40 \mathrm{~nm}$ ). Top trace, The outward photocurrent elicited by the excitation light, measured at a holding potential of $-30 \mathrm{mV}$. Bottom trace, The emitted fluorescence ( $\lambda_{\max }=535 \mathrm{~nm}$; bandwidth, $50 \mathrm{~nm}$ ), collected from a $10 \times 10 \mu \mathrm{m}$ region that included the light-sensitive ciliary appendages of the cell. The optical signal jumped abruptly after the shutter was opened, but no additional change in fluorescence occurred during the light response. Inset, A similar experiment conducted in a microvillar (depolarizing) cell from the same preparation. In this case, the photocurrent was inwardly directed, and the fluorescence signal measured from the light-sensitive lobe indicated a pronounced $\mathrm{Ca}^{2+}$ increase coincident with the light response. cps, Counts per second.

sponse. This contrasts sharply with similar experiments conducted in microvillar cells from the same preparation, as shown in the inset in Figure 1. In this case, the photocurrent was inwardly directed (consistent with the fact that, in these cells, the receptor potential is depolarizing), and the fluorescence signal measured from the light-transducing lobe of the cell reported a pronounced $\mathrm{Ca}^{2+}$ increase coincident with the light response, similar to that observed in microvillar photoreceptors in other species. Three additional ciliary photoreceptors tested in the same manner also failed to reveal any detectable Ca changes. To assess whether there may be $\mathrm{Ca}$ transient of low amplitude, which may be missed by the $5 \mathrm{~N}$ Ca probe, we also tested a calcium indicator of much higher affinity (Calcium Green-2; $K_{\mathrm{D}}$ of 0.55 $\mu \mathrm{M})$ and again failed to detect any light-induced change in $\left[\mathrm{Ca}^{2+}\right]_{\mathrm{i}}(n=2)$. Repetition of the measurement after a darkadaptation interval produced a similar fluorescence level, indicating that slower changes occurring after the sampled temporal window are unlikely.

The failure to detect any photo-induced changes in intracellular calcium within the operating range at which ciliary photoreceptors undergo light adaptation prompted us to look for alternative pathways that could modulate the transduction cascade. An upregulation of a phosphodiesterase would be a plausible possibility, but the application of PDE inhibitors (both broad spectrum, such as IBMX, as well as those selective for cGMPspecific PDE, such as dipyridamole) failed to alter the peak-toplateau decay of the photocurrent evoked by a prolonged light step (data not shown). In contrast, cGMP, the messenger that also signals visual excitation, emerged as a candidate. The key clue was that, after activation of the light-sensitive $\mathrm{K}^{+}$current by internal dialysis with cGMP or analogs thereof, the light response was substantially depressed (Gomez and Nasi, 1995). Qualitatively, one would, of course, expect such a result on the assumption that the two stimuli (light and cGMP) tap the same effector mechanism. However, from the quantitative point of view, a simple competition scheme predicts that the observed reduction in the amplitude of the light response should be accounted for by the

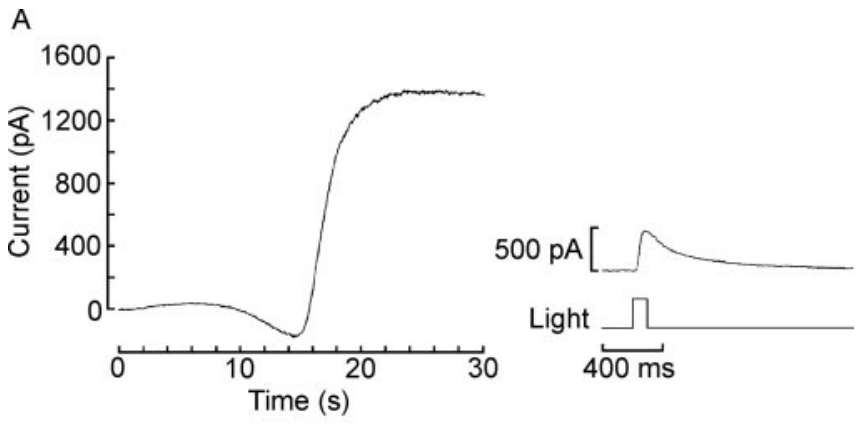

B

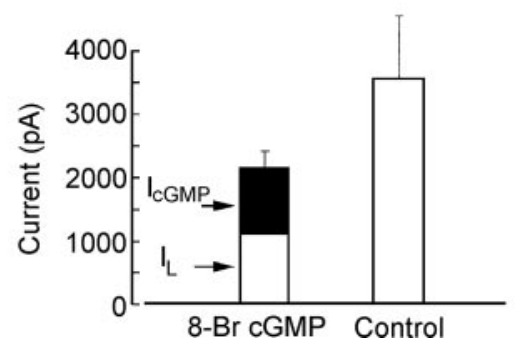

Figure 2. Stimulation of the light-sensitive conductance by GMP antagonizes the light response. $A$, Left, Outward potassium current activated by internal perfusion with $20 \mu \mathrm{m} 8-\mathrm{Br}$ cGMP in the dark. The trace begins at the time the membrane patch was ruptured to gain access to the intracellular compartment. Right, After equilibration, a 100 ms flash of saturating intensity was delivered and elicited a photoresponse of only $\approx 500 \mathrm{pA}$. B, Summary of the results pooled from five control cells and four cells treated with 8-Br-cGMP: the bar on the left represents the sum of the CGMP-evoked current (filled portion) and the residual photocurrent (open portion); the total falls significantly short of the maximum amplitude of the light-evoked current measured in control cells (right). Error bars indicate SD.

size of the cyclic nucleotide-activated current. In other words, the magnitude of the residual photoresponse should reflect the reduced pool of ion channels that remain available to respond to photostimulation. Instead, it seemed that, even under conditions that elicited a cGMP-dependent current of a few hundred picoamperes (i.e., 10-20\% the size of the maximal photocurrent), the attenuation of the light response was dramatic. We sought to clarify this issue by systematically examining the amplitude of the cGMP-dependent membrane current in the dark and that of the current subsequently evoked by flashes of light. Figure 2 shows a typical recording from a ciliary photoreceptor, immediately after gaining access to the cell interior and initiating internal dialysis with $20 \mu \mathrm{M}$ 8-Br-cGMP. An outward current was activated within seconds, reaching an amplitude of $\sim 1.5 \mathrm{nA}$. After stabilization, subsequent photostimulation with a saturating light intensity elicited a response of only $\approx 500 \mathrm{pA}$ (Fig. $2 A$, right). We compared the combined current in response to photostimulation and to cGMP analogs with the light-evoked current under control conditions. The bar graph in Figure $2 B$ summarizes the results pooled from five control cells and four cells treated with 8-Br-cGMP: the bar on the left represents the sum of the cGMPevoked current (dark portion) and the residual photocurrent (clear portion); it is evident that the total falls significantly short of the maximum amplitude of light-evoked current obtained in control cells (right), which is of several nanoamperes $(p<0.05$; $t$ test).

The above observations suggest that, in addition to stimulating the light-dependent conductance, cGMP or some mechanism located farther downstream in the cascade depresses the phototransduction. To examine further how the effects of cGMP analogs are akin to those of light adaptation, intensity series were recorded from photoreceptors dialyzed with control intracellular 
A

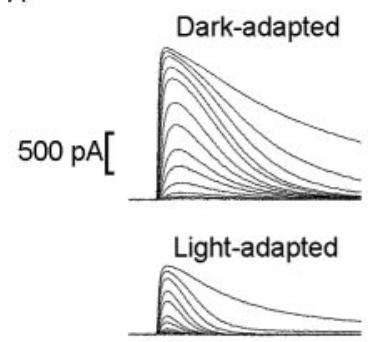

Light_L $4 \longdiv { 0 0 \mathrm { ms } }$

B
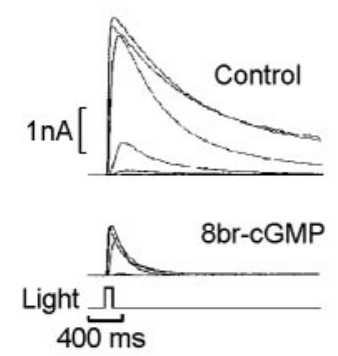

C
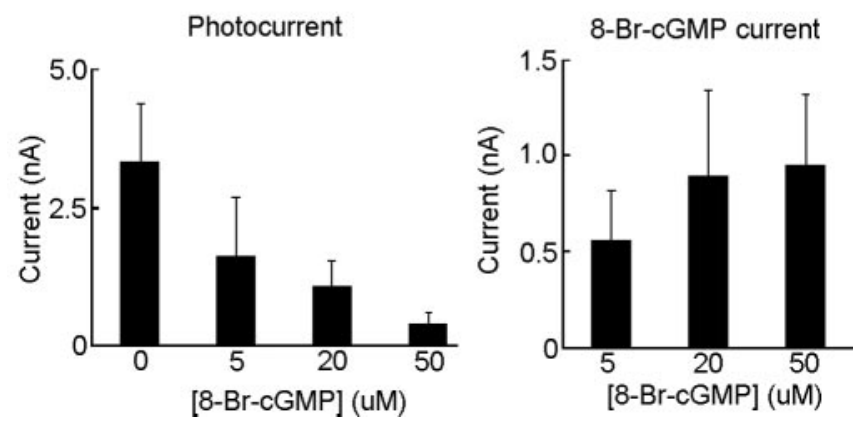

Figure 3. cGMP decreases light sensitivity. A, Left, Intensity series recorded from a control photoreceptor in the dark-adapted (D.A.) state (top) and in the presence of an adapting background light $\left(4.2 \times 10^{14}\right.$ photons $\left.\cdot \mathrm{cm}^{-2} \cdot \mathrm{s}^{-1}\right)$. The peak amplitudes of the photocurrent are plotted as a function of test flash intensity on the right. The $20 \mathrm{~ms}$ unattenuated flash delivered $1.62 \times 10^{14}$ photons $\times \mathrm{cm}^{-2}$ (photon flux, $1.29 \mathrm{log}$ relative to the background). L.A., Lightadapted. $\boldsymbol{B}$, Effect of 8-Br-cGMP on the intensity-response curve. The top family of traces was measured in a control cell, and the bottom family was measured in a different photoreceptor internally dialyzed with $20 \mu \mathrm{m} 8$ 8-Br-cGMP. Right, Average peak amplitude of the photocurrents measured under the two conditions, plotted as a function of light intensity $(n=4$ and 5 , respectively); error bars indicate SD. Unattenuated test flash, $1.38 \times 10^{14}$ photons $\times \mathrm{cm}^{-2}$.C, Left, Maximum amplitude of the photocurrent measured with control intracellular solution and with increasing concentrations of 8- $\mathrm{Br}-\mathrm{CGMP}(n=6,5,4$, and 4). Right, Magnitude of the membrane currents evoked in the dark by internal perfusion of the same concentrations of 8-Br-CGMP.

solution or with $20 \mu \mathrm{M} 8$-Br-cGMP. Figure $3 A$ illustrates typical intensity series measured in a control cell under dark-adapted versus background-adapted conditions; on the right, the corresponding peak photocurrent amplitudes are plotted as a function of light intensity. The compression of the response range and right shift of the intensity-response curve are readily observed. Figure $3 B$ shows light-evoked currents from one control cell and one dialyzed with $20 \mu \mathrm{M} 8$-Br-cGMP. On the right, the average peak amplitude of the photocurrents measured under the two conditions is plotted as a function of light intensity $(n=4$ and 5, respectively). The cGMP analog also reduced response ampli-
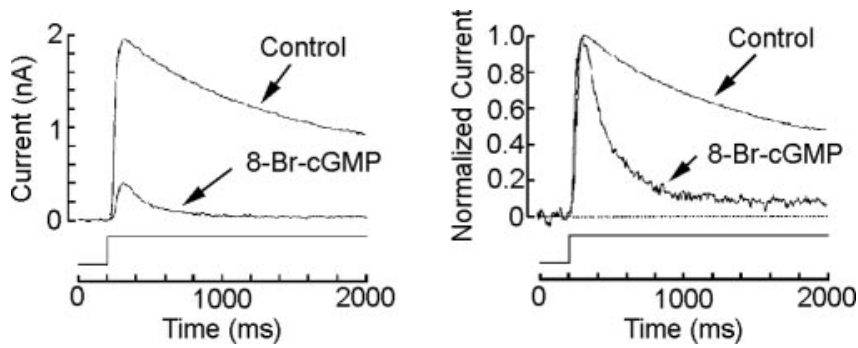

Figure 4. cGMP analogs potentiate light-induced desensitization. Ciliary photoreceptor cells voltage clamped at $-30 \mathrm{mV}$ were stimulated with a sustained light stimulus sufficiently bright to induce adaptation $\left(8.8 \times 10^{14}\right.$ photons $\left.\cdot \mathrm{cm}^{-2} \cdot \mathrm{s}^{-1}\right)$. Left, Superimposed photocurrents measured in one control dark-adapted cell and in one dialyzed with $20 \mu \mathrm{m} 8$ 8-Br-cGMP; the amplitude of the response was markedly reduced in the treated cell. Right, The same two records have been normalized with respect to the peak, highlighting the pronounced acceleration in the relaxation and the reduced steady-state plateau after administration of the cGMP analog.

tudes and shifted sensitivity, in a manner reminiscent of the effects of background illumination. The desensitization produced by 8 -Br-cGMP was dose dependent. The bar graph in Figure $3 C$ (left) shows the mean saturating amplitude of the photocurrent measured in control conditions and after dialysis with 5, 20, and $50 \mu \mathrm{M} 8$ 8-Br-cGMP, respectively. Light responsiveness decreased monotonically with concentration within this range. On the right, the average amplitudes of the current directly evoked by the cGMP analog are displayed.

If cGMP partakes of the signaling pathway for light adaptation, one would anticipate a synergistic interaction between adapting illumination and the exogenous administration of cGMP analogs. We examined the effects of 8 -Br-cGMP on the peak-to-plateau decay of the response evoked by a sustained light stimulus, another typical measure of light adaptation. Figure 4 (left) shows the photocurrents evoked by prolonged light steps in one control cell and one internally dialyzed with $50 \mu \mathrm{M} 8$-BrcGMP. In the latter, not only was responsiveness markedly depressed, but the falling phase of the response is substantially more pronounced (reminiscent of the rapid decay one observes with brighter light steps); on the right, the same two traces were normalized with respect to the peak amplitude, highlighting the reduced relative amplitude of the plateau and the more rapid relaxation in the presence of 8 -Br-cGMP $(n=3)$.

A characteristic concomitant of light adaptation is the acceleration of the flash response, as illustrated in Figure $5 \mathrm{~A}$ (left): the photocurrent evoked by a subsaturating test stimulus was recorded in dark-adapted conditions and superimposed on an adapting background. In addition to the depression in amplitude, the response was noticeably accelerated, as best appreciated in Figure $5 A$ (right), in which the responses were normalized. Similarly, the effects of cGMP stimulation on the photocurrent were not limited to sensitivity but also altered the time course of the photocurrent. Figure $5 B$ shows superimposed light-evoked currents elicited by a test flash of fixed, subsaturating intensity, recorded in one control photoreceptor and one dialyzed with 20 $\mu \mathrm{M} 8$ - $\mathrm{Br}$-cGMP; the dramatic difference in time course is highlighted on the right, in which the light responses were normalized with respect to the peak. To quantify this effect, we measured the width of the photocurrent at full-width half-maximal (FWHM) amplitude. We found that, even with concentrations as low as 5 $\mu \mathrm{M}$, the response was greatly accelerated (FWHM, $169 \pm 28$ vs $432 \pm 71 \mathrm{~ms}$ in controls; $n=5$ and 6 , respectively; $p<0.0001 ; t$ test). As shown in Figure $5 C$, no significant additional accelera- 
A
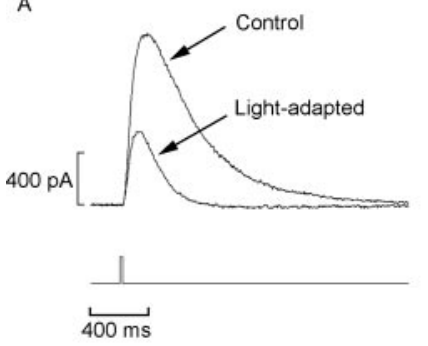

B
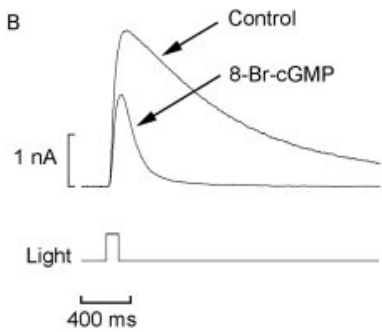

C

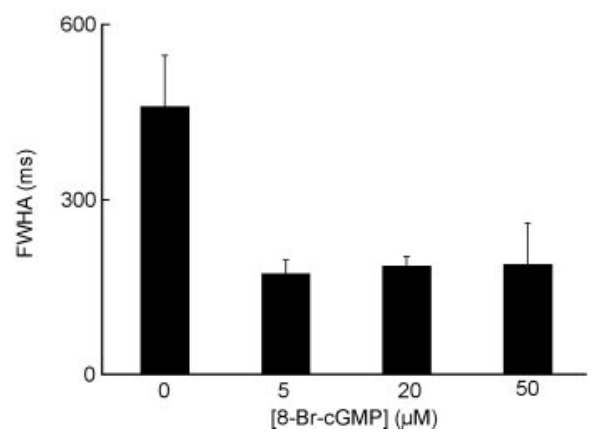

Figure 5. Acceleration of the photocurrent by $8-\mathrm{Br}-\mathrm{cGMP}$ resembles light adaptation effects. $\boldsymbol{A}$, Left, Superimposed flash responses $\left(4.95 \times 10^{13}\right.$ photons $\left.\times \mathrm{cm}^{-2}\right)$ in a control photoreceptor stimulated under dark-adapted conditions and subsequently during exposure to sustained background illumination $\left(4.2 \times 10^{13}\right.$ photons $\left.\cdot \mathrm{cm}^{-2} \cdot \mathrm{s}^{-1}\right)$. Right, The same two traces were normalized with respect to their peak amplitude (Norm. Ampl.), revealing a pronounced acceleration in response kinetics after light adaptation. $\boldsymbol{B}, \mathrm{A}$ similar comparison is made between the dark-adapted responses in one control cell and one dialyzed with $20 \mu \mathrm{m}$ 8-Br-cGMP. Again, the treatment caused not only a reduction in photocurrent amplitude but also a more rapid time course. C, Flash response speed, measured as the FWHM of the lightevoked current, in control conditions and during perfusion with increasing concentrations of 8-Br-cGMP, in separate groups of cells. Error bars denote SD.

tion occurred after increasing the concentration of 8-Br-cGMP to 20 or $50 \mu \mathrm{M}$. The changes in response kinetics induced by light adaptation and by 8 -Br-cGMP did not differ significantly. The normal form of cGMP produced qualitatively similar effects but required higher concentrations ( $>1 \mathrm{~mm}$; data not shown). In summary, although the possibility of additional pharmacological effects of 8-Br-cGMP cannot be ruled out entirely, the evidence indicates that the stimulation of the cGMP pathway desensitizes and accelerates the light response, similar to what background illumination does, and potentiates the effects of light adaptation.

The next question concerns the mechanisms by which cGMP may act to reduce light responsiveness. In addition to its direct role in the gating of certain ionic channels, cGMP has been implicated in the modulation of a variety of cellular processes via its action on regulatory proteins. Among the best characterized downstream effectors of cGMP is PKG, which phosphorylates threonine and serine residues in a variety of target proteins (for review, see Wang and Robinson, 1997). We ascertained the pos- sible involvement of this pathway by testing the ability of PKG inhibitors to interfere with the process of light adaptation. The protocol consisted of a test flash presented initially under darkadapted conditions and subsequently in the presence of a $7 \mathrm{~s}$ light step. The onset of the background illumination preceded the test flash by several seconds, to allow the sustained photocurrent to attain a near-steady state before eliciting an incremental response. Two background intensities were used, differing by 1 log unit. Figure $6 A$ shows representative examples of pairs of recordings (dark- and light-adapted with the same dim background intensity) in a control photoreceptor and one internally dialyzed with $3 \mu \mathrm{M} \mathrm{KT5823}$; in the presence of the PKG inhibitor, the incremental response was less depressed by the background. To quantify the effect, the ratio of the light-adapted response amplitude divided by the dark-adapted response amplitude was compared in the two conditions; the difference was statistically significant for both the dimmer adapting light $(p<0.01 ; t$ test; $n=9$ and 11 for experimental and control cells, respectively) and the brighter background ( $p<0.005$ ). The background-induced acceleration of the flash response was also reduced in the presence of the PKG inhibitor ( $p<0.02$ for both intensities). The data are summarized in Figure $6 B$ : the bar graph shows that, for each condition, both parameters (namely, relative amplitude and relative FWHM of the incremental response) were larger in cells treated with KT5823. A more extended protocol, in which the intensity of the adapting background was progressively increased over a range spanning $3.6 \mathrm{log}$ units, could be completed in a smaller subset of cells $(n=4)$. Figure $6 C$ shows the pooled data, plotted as a function of the adapting background intensity; in the presence of KT5823, the normalized size of the incremental response remained larger than in control cells over a substantial portion of the adapting range. Although there was a hint that KT5823 may have increased responsiveness in fully dark-adapted cells, a comparison of basal parameters of the light response (amplitude, sensitivity, and kinetics) failed to attain statistical significance $(n=9$ and 11 for KT5823-treated and control cells, respectively). The concentration of KT5823 used should be saturating for PKG and approaches the range in which this compound may also inhibit PKC; however, control experiments using specific manipulations of PKC were completely ineffective (Piccoli et al., 2002), so the observed effects should be attributable to PKG. We attempted similar experiments with a synthetic peptide (G-1458) derived from the PKG substrate histone H2B, which is designed to serve as a competitive pseudosubstrate. Although the trend was comparable with the effects of KT5823, in that for each intensity of background illumination the mean desensitization was attenuated with respect to control cells, the comparison fell short of statistical significance $(n=4)$. Overall, the experiments using the peptide yielded more variability, perhaps because the effectiveness of the dialysis is compromised not only by the its relatively large mass but also its susceptibility to proteolytic degradation and adsorption to vessel surfaces [an effect that we tried to alleviate by the use of BSA as a carrier protein (Suelter and DeLuca, 1983)]. An additional agent, DT-3, has recently been proposed to act as a membrane-permeable inhibitor of PKG, but local extracellular application at a concentration of 5 $\mu \mathrm{M}$ did not significantly alter the response to prolonged light steps (data not shown).

Despite the use of saturating concentrations of KT5823, we never observed a complete reversal to the dark-adapted response phenotype. In other words, in the presence of the inhibitor, the adapting background still induced a marked desensitization and response acceleration. This incomplete effectiveness may be in- 
A
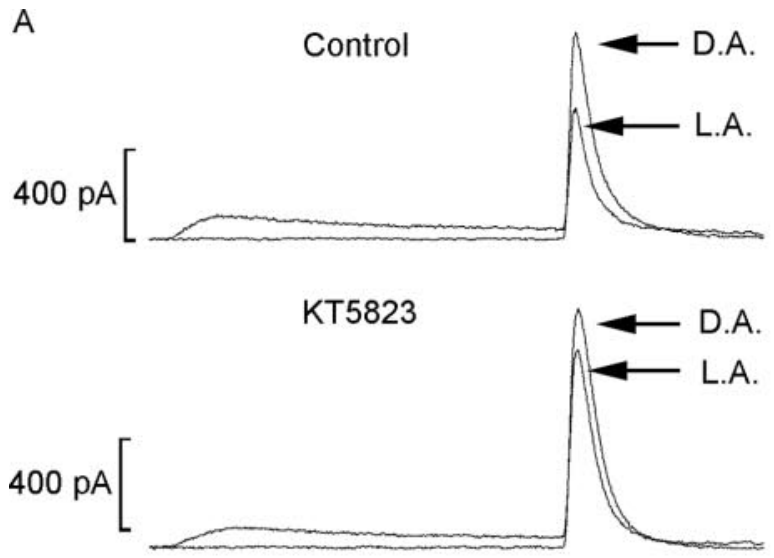

Test

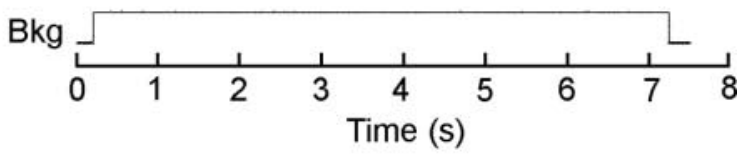

B
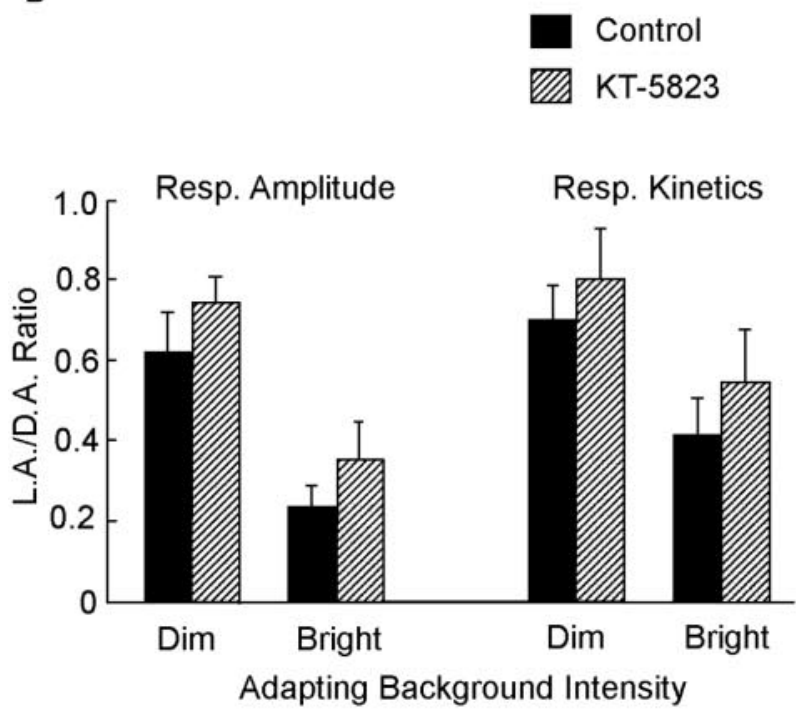

C

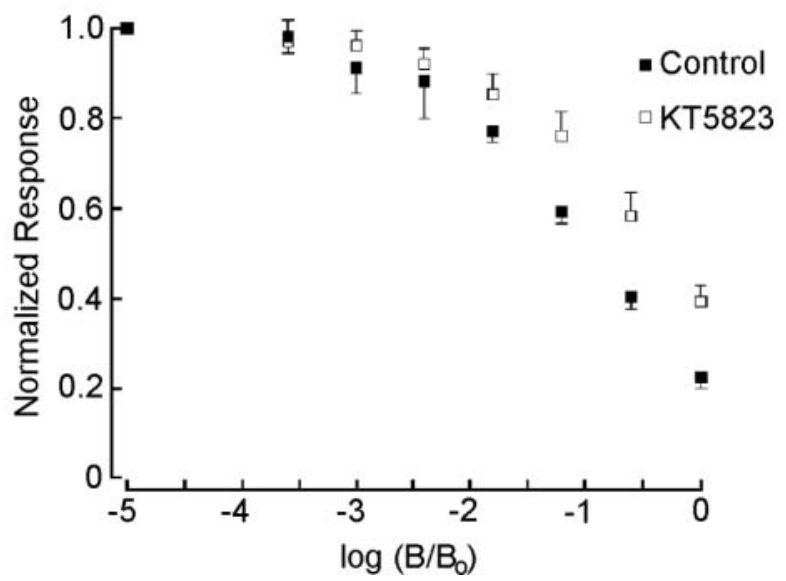

Figure 6. Desensitization is reduced by an inhibitor of PKG. $A$, The response (Resp.) to a test flash of fixed, just-saturating intensity was measured under dark-adapted (D.A.) conditions and in the presence of a steady background $(\mathrm{Bkg})$ light $\left(5.33 \times 10^{12}\right.$ photons $\left.\cdot \mathrm{cm}^{-2} \cdot \mathrm{s}^{-1}\right)$, which was turned on $4.8 \mathrm{~s}$ before the test flash $\left(5.6 \times 10^{13}\right.$ photons $\left.\times \mathrm{cm}^{-2}\right)$. The top traces dicative of the limitations of the pharmacological agents used or, alternatively, may point to additional signals for light adaptation. A clue concerning a potential regulator of response speed had surfaced in a previous study on the role of cGMP as a messenger for visual excitation: we observed that the direct chemical activation of the light-dependent conductance was strictly nucleotide dependent, and adenine nucleotides were completely ineffective (Gomez and Nasi, 1995). Nevertheless, the shape of the lightevoked current seemed to be visibly influenced by application of 8-Br-cAMP. We revisited this issue by examining more systematically various manipulations designed to target the cAMP pathway. Figure 7 shows two light-intensity series measured in a control cell and in a cell subjected to internal perfusion with $50 \mu \mathrm{M}$ 8 -Br-cAMP for several minutes. The most conspicuous effect is a dramatic acceleration of the photocurrent kinetics, especially the decay phase; this phenomenon seems particularly striking in Figure $7 C$, which shows the superimposed, normalized responses to a flash of approximately half-saturating intensity delivered in the two conditions. As a quantitative index of the response time course, we again used the full width measured at half-maximal amplitude. The bar graph in Figure $7 D$ shows the mean value for eight control cells and nine cells dialyzed with $50 \mu \mathrm{M} 8$ 8-Br-cAMP; the difference between the two conditions was statistically significant ( $p<0.02 ; t$ test). We tried higher doses of the compound $(250 \mu \mathrm{M})$, but the accelerating effects were not more pronounced $(n=4$; data not shown). In contrast, as shown in Figure $7 B$, there was no statistically significant reduction in the amplitude of the flash response in the presence of 8-Br-cAMP $(p>0.25)$; furthermore, the light intensity needed to evoke a half-saturating response $(\sigma)$ was also not significantly different from that of control cells. The natural, hydrolyzable form of cAMP produced a similar acceleration of the photoresponse, although the concentrations required were in the millimolar range (data not shown).

\section{Discussion}

Ciliary invertebrate photoreceptors lack the two chief mechanisms that, in other visual cells, inextricably link light stimulation to changes in cytosolic calcium: first, the light-sensitive channels are not measurably permeable to Ca (Nasi and Gomez, 1999a); second, the phototransduction cascade seems to not use the $\mathrm{G}_{\mathrm{q}}$ / PLC pathway for signaling (Gomez and Nasi, 1995, 2000; Kojima et al., 1997), and, thus, Ca release from $\mathrm{IP}_{3}$-sensitive internal stores is unlikely. To rule out the possibility of other, unanticipated sources of photo-induced changes in cytosolic calcium, we directly monitored intracellular $\left[\mathrm{Ca}^{2+}\right]$ using fluorescent indicators spanning a wide range of affinities $\left(K_{\mathrm{D}}\right.$ of $\left.0.55-14 \mu \mathrm{M}\right)$. Our results corroborate the notion that, in sharp contrast with microvillar photoreceptors present in the same retina (as well as

\footnotetext{
$\leftarrow$

were recorded with standard intracellular solution, whereas the bottom pair were obtained from a different photoreceptor dialyzed with $3 \mu \mathrm{m}$ KT5823. L.A., Light-adapted. B, Summary of the effects of KT5823 on background adaptation parameters. The peak amplitude and the FWHM of the incremental response, relative to dark-adapted conditions, were averaged for 11 control cells (filled bars) and nine cells dialyzed with KT5823 (cross-hatched bars). Both measurements indicate that, in each case, the PKG inhibitor partially antagonized the effects of the adapting light. The dimmer background was as in $\boldsymbol{A}$; the brighter background was increased by 1 log unit. Error bars indicate SD. C, Effects of varying the intensity of the adapting background. The mean amplitude of the flash response (normalized with respect to that measured in darkadapted conditions) is plotted as a function of the intensity of the background light, which was raised at $0.6 \log$ increments (unattenuated intensity, $5.1 \times 10^{14}$ photons $\cdot \mathrm{cm}^{-2} \cdot \mathrm{s}^{-1}$ ). The experiment was conducted in five control cells and four cells treated with KT5823; error bars denote SD.
} 
A

Control

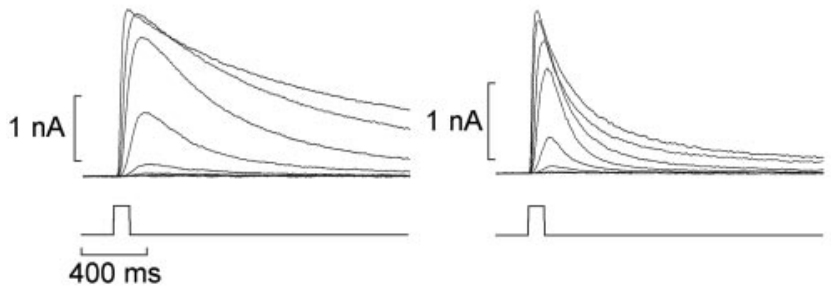

B Maximun Current Amplitude

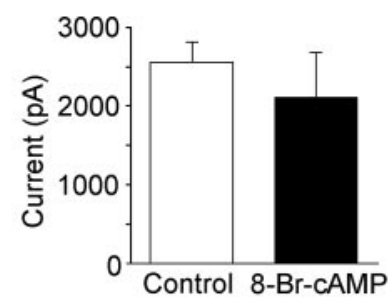

C

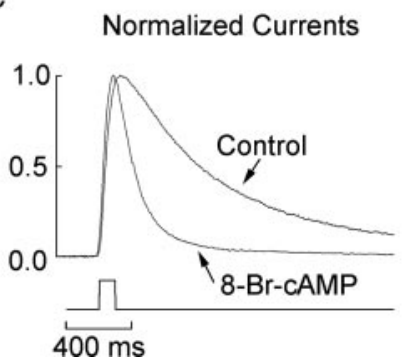

D

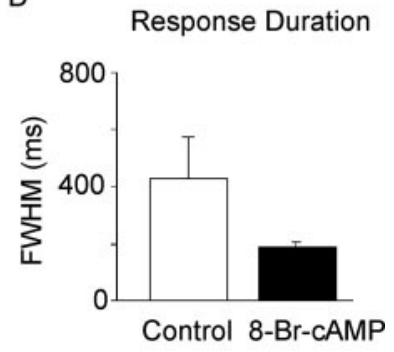

Figure 7. Modulation of response kinetics by cAMP analogs. $\boldsymbol{A}$, Representative intensity series obtained in one voltage-clamped control cell (left) and one dialyzed with $50 \mu \mathrm{m} 8-\mathrm{Br}$ cAMP (right). Test flashes were $100 \mathrm{~ms}$ in duration, and the holding potential was $-30 \mathrm{mV}$. $\boldsymbol{B}$, Mean amplitude of the saturating photocurrent for control and 8-Br-cAMP-treated cells; the difference was not statistically significant. Error bars indicate SD. C, Normalized, superimposed traces of photocurrents elicited by flashes of identical, subsaturating intensity. The time course of the response obtained in the presence of 8-Br-cAMP is markedly accelerated. $\boldsymbol{D}$, Bar graph representing the average width of the flash response at half-maximal amplitude ( $n=8$ and 9 for control and 8-Br-cAMP-treated cells, respectively).

those found in other species), the light response of ciliary photoreceptors is wholly decoupled from any detectable change in $[\mathrm{Ca}]_{\mathrm{i}}$, at least over the duration of the interval monitored (500 $\mathrm{ms}$ ), which is substantially longer than the time constant for adaptation to set in (Gomez and Nasi, 1997). It is true that these cells are capable of generating Ca-dependent action potentials (Cornwall and Gorman, 1979), but those occur at the termination of a light stimulus and are responsible for triggering the well known defensive "shadow reflex" (Hartline, 1938). No contribution of depolarization-activated calcium channels in altering cytosolic $\mathrm{Ca}$ is therefore expected in the dark (because of steady-state inactivation at $V_{\text {rest }}$, which is approximately $-35 \mathrm{mV}$ ), nor during illumination, when the membrane potential hyperpolarizes. In conjunction with previous studies in which we reported that exogenous administration of Ca chelators and buffered Ca had little effect either on the photocurrent (Gomez and Nasi, 1995) or on light adaptation (Gomez and Nasi, 1997), the present results argue that ciliary photoreceptors are indeed unique, in that they dispense altogether with calcium as a key regulator of the visual process.

However, these cells exhibit a normal susceptibility to adapt- ing lights (Gomez and Nasi, 1997), which implies that Caindependent modulatory mechanisms of the phototransduction cascade must be operative. A clue toward the elucidation of possible signaling pathways for adaptation was provided by the effects of intracellular administration of cGMP and 8-Br-cGMP. These substances activate the light-sensitive K conductance and, concomitantly, depress the light response (Gomez and Nasi, 1995). The reduction in the size of the photocurrent, however, significantly exceeds the amount expected from a simple occlusive interaction in which light and cGMP stimulation compete for a common pool of ion channels and suggests instead that this nucleotide must also downregulate, on a slower time scale, the light response. cGMP seems to mimic the phenotype of light adaptation, including (1) a compression of the response amplitude range, (2) a right shift of the stimulus-response curve, (3) a marked acceleration of the light response time course, and (4) a synergistic interaction with adapting background illumination. As such, cGMP seems to perform a double function, serving as a signal for both visual excitation and adaptation. This dual action of cGMP is somewhat reminiscent of the effects of calcium in microvillar invertebrate photoreceptors, in which a lightinduced elevation of $[\mathrm{Ca}]_{\mathrm{i}}$ is critical for boosting the gain of the phototransduction cascade and also triggers a downregulation of its sensitivity (Payne and Fein, 1986; Payne et al., 1986; Hardie, 1995); obviously, these two processes must operate at different time scales and via different effectors. An even more germane precedent for the use of the same messenger for excitation and sensory adaptation has been reported in the cilia of rat olfactory neurons, in which a class of odorants stimulate adenylyl cyclase, and the resulting elevation of cAMP (the substance responsible for gating the receptor current) activates PKA, which in turn feeds back negatively onto cAMP production (Boekhoff and Breer, 1992). The mechanism(s) by which cGMP helps control the state of light adaptation of ciliary photoreceptors remain to be fully elucidated. Our observation that the background lightinduced desensitization of the photocurrent is attenuated by intracellular administration of the PKG inhibitor KT5823 suggests that a cGMP-dependent protein kinase may participate in the negative-feedback regulation governed by cGMP; the possible targets for phosphorylation are yet to be identified. Sequence analysis algorithms applied to two molecularly identified elements of the transduction cascade, the rhodopsin and the $G_{0}$ (Kojima et al., 1997), failed to predict sites for PKG phosphorylation; however, other potential candidates are the guanylate cyclase, presumed to bring about a light-dependent increase in cGMP, and the light-sensitive ion channels.

One of the hallmarks of light adaptation is the acceleration of the time course of the photocurrent, a process that optimizes responsiveness by trading speed for sensitivity. 8-Br-cGMP mimics this phenomenon but with a concentration dependency that differs from its effects on sensitivity (compare Figs. 3C, 5C). One interpretation is that these two attributes of light adaptation could be controlled separately by cGMP; for example, we reported previously that, after termination of an adapting stimulus, the amplitude of the photocurrent can recover more rapidly than its time course (Nasi and Gomez, 1999b). We reported recently a similar dissociation in microvillar photoreceptors, in which light-dependent activation of PKC participates in the process of sensory adaptation, but its role may be circumscribed to the desensitization of the photoresponse and not the modulation of its kinetics (Piccoli et al., 2002). In ciliary photoreceptors, cAMP and its slowly hydrolyzable analog 8-Br-cAMP accelerate the light response in a way similar to that of background light and cGMP 
but without significantly attenuating response sensitivity. In one interpretation of these observations, cAMP could emerge as a candidate downstream effector of cGMP to help modulate the time course of the light response. This tantalizing possibility would require cross-talk between cGMP and cAMP signaling, which has been described in a variety of systems. An enzyme that could fulfill such a role is PDE III (Beavo, 1995), because it is inhibited by cGMP and uses cAMP as a specific substrate. However, exploratory tests of this proposition yielded inconclusive results: neither the PDE III antagonist cilostamide ( $1 \mu \mathrm{M} ; n=3)$ nor the protein kinase A inhibitor Rp cAMP (100 $\mu \mathrm{M} ; n=4)$ interfered with the response acceleration produced by background illumination. Finally, we attempted to stimulate an elevation of cAMP with $20 \mu \mathrm{M}$ forskolin, an activator of adenylyl cyclase, but we observed no speeding of the photocurrent with either extracellular or intracellular application $(n=4$ in each condition). Little is known about the chemistry/pharmacology of these enzymes in mollusks, and it is therefore conceivable that different isoforms, less susceptible to these drugs, may be involved. Alternatively, a more parsimonious account could be that cAMP acts on the same substrate at which cGMP controls response acceleration with high apparent affinity but is a poor substitute for cGMP in the modulation of sensitivity (and the gating of the photoconductance). Regardless of the interpretation, these distinct aspects of sensory adaptation seem to be separately controlled. Ciliary photoreceptors provide a uniquely favorable setting to dissect Ca-independent modulatory mechanisms of visual function, which may prove relevant to other sensory cells that use cyclic-nucleotide-based signaling (Gray-Keller and Detwiler, 1996).

\section{References}

Bader CR, Baumann F, Bertrand D (1976) Role of intracellular calcium and sodium in light adaptation on the retina of the honey bee drone (Apis mellifera, L.). J Gen Physiol 67:475-491.

Beavo J (1995) Cyclic nucleotide phosphodiesterases: functional implications of multiple isoforms. Physiol Rev 75:725-748.

Boekhoff I, Breer H (1992) Termination of second messenger signaling in olfaction. Proc Natl Acad Sci USA 89:471-474.

Brown JE, Blinks JR (1974) Changes in intracellular free calcium concentration during illumination of invertebrate photoreceptors. J Gen Physiol 64:643-665.

Cornwall MC, Gorman AL (1979) Contribution of calcium and potassium permeability changes to the off response of scallop hyperpolarizing photoreceptors. J Physiol (Lond) 291:207-232.

Cornwall MC, Gorman AL (1983) Ionic and spectral mechanisms of the off response to light in hyperpolarizing photoreceptors of the clam, Lima scabra. Cell Mol Neurobiol 3:311-328.

Fain GL, Matthews HR, Cornwall MC, Koutalos Y (2001) Adaptation in vertebrate photoreceptors. Physiol Rev 81:117-151.

Fein A, Charlton JS (1977) A quantitative comparison of the effects of intracellular calcium injection and light adaptation on the photoresponse of Limulus ventral photoreceptors. J Gen Physiol 70:591-600.

Gomez M, Nasi E (1994) The light-sensitive conductance of hyperpolarizing invertebrate photoreceptors: a patch-clamp study. J Gen Physiol 103:939-956.

Gomez M, Nasi E (1995) Activation of light-dependent potassium channels in ciliary invertebrate photoreceptors involves cGMP but not the $\mathrm{IP}_{3} / \mathrm{Ca}$ cascade. Neuron 15:607-618.

Gomez M, Nasi E (1997) Light adaptation in Pecten hyperpolarizing photoreceptors: insensitivity to Ca manipulations. J Gen Physiol 109:371-384.

Gomez M, Nasi E (1999) Calcium-independent, cGMP-mediated desensi- tization of the light response in Pecten ciliary photoreceptors. Biophys J 76:A243.

Gomez M, Nasi E (2000) Light transduction in invertebrate hyperpolarizing photoreceptors: involvement of a $\mathrm{G}_{\mathrm{o}}$-regulated guanylate cyclase. J Neurosci 20:5254-5263.

Gorman ALF, McReynolds JS (1978) Ionic effects on the membrane potential of the hyperpolarizing photoreceptors in scallop retina. J Physiol (Lond) 275:345-355.

Gray-Keller MP, Detwiler PB (1996) $\mathrm{Ca}^{2+}$ dependence of dark- and lightadapted flash responses in rod photoreceptors. Neuron 17:323-331.

Hardie RC (1995) Photolysis of caged $\mathrm{Ca}^{2+}$ facilitates and inactivates but does not directly excite light-sensitive channels in Drosophila photoreceptors. J Neurosci 15:889-902.

Hardie RC, Peretz A, Suss-Toby E, Rom-Glas A, Bishop SA, Selinger Z, Minke B (1993) Protein kinase C is required for light adaptation in Drosophila photoreceptors. Nature 363:634-637.

Hartline HK (1938) The discharge of impulses in the optic nerve of Pecten in response to illumination of the eye. J Cell Comp Physiol 2:465-478.

Kojima D, Terakita A, Ishikawa T, Tsukahara Y, Maeda A, Shichida Y (1997) A novel $\mathrm{G}_{\mathrm{o}}$-mediated phototransduction cascade in scallop visual cells. J Biol Chem 272:22979-22982.

Lisman JE, Brown JE (1972) The effects of intracellular iontophoretic injection of calcium and sodium ions on the light response of Limulus ventral photoreceptors. J Gen Physiol 59:701-719.

Lisman JE, Brown JE (1975) Effects of intracellular injection of calcium buffers on light adaptation in Limulus ventral photoreceptors. J Gen Physiol 66:489-506.

McNaughton PA, Cervetto L, Nunn BJ (1986) Measurement of the intracellular free calcium concentration in salamander rods. Nature 322:261-263.

McReynolds JS (1976) Hyperpolarizing photoreceptors in invertebrates. In: Neural principles in vision (Zettler F, Weiler R, eds) pp 394-409. Berlin: Springer.

Nasi E, Gomez M (1999a) Divalent cation interactions with lightdependent $\mathrm{K}$ channels: kinetics of voltage-dependent block and requirement for an open pore. J Gen Physiol 114:653-671.

Nasi E, Gomez M (1999b) Dissociation of kinetics and desensitization effects of light adaptation in ciliary photoreceptors of Pecten. Biophys J 76:A243.

Payne R, Fein A (1986) The initial response of Limulus ventral photoreceptors to bright flashes: released calcium as synergist to excitation. J Gen Physiol 87:243-269.

Payne R, Corson DW, Fein A (1986) Pressure injection of calcium both excites and adapts Limulus ventral photoreceptors. J Gen Physiol $88: 107-126$.

Payne R, Flores TM, Fein A (1990) Feedback inhibition by calcium limits the release of calcium by inositol trisphosphate in Limulus ventral photoreceptors. Neuron 4:547-555.

Piccoli G, Gomez M, Nasi E (2002) Role of protein kinase C in light adaptation of microvillar photoreceptors. J Physiol (Lond) 543:481-494.

Ratto GM, Payne R, Owen WG, Tsien RY (1988) The concentration of cytosolic free calcium in vertebrate rod outer segments measured with fura-2. J Neurosci 8:3240-3246.

Smith DP, Ranganathan R, Hardy RW, Marx J, Tsuchida T, Zuker CS (1991) Photoreceptor deactivation and retinal degeneration mediated by a photoreceptor-specific protein kinase C. Science 254:1478-1484.

Suelter CH, DeLuca M (1983) How to prevent losses of protein by adsorption to glass and plastic. Anal Biochem 135:112-119.

Wang W, Robinson PJ (1997) Cyclic GMP-dependent protein kinase ands cellular signaling in the nervous system. J Neurochem 68:443-456.

Yau KW, Nakatani K (1984a) Cation selectivity of light-sensitive conductance in retinal rods. Nature 309:352-354.

Yau KW, Nakatani K (1984b) Electrogenic Na-Ca exchange in retinal rod outer segments. Nature 311:661-663.

Yau KW, Nakatani K (1985) Light-induced reduction of cytoplasmic free calcium in retinal rod outer segment. Nature 313:579-582. 